\title{
Postoperative Cognitive Dysfunction following General Anaesthesia in Patients Undergoing Elective Non-cardiac Surgery
}

\author{
Muhammad Saad Yousuf, Khalid Samad and Hameed Ullah \\ Department of Anaesthesiology, The Aga Khan University Hospital, Karachi, Pakistan
}

\begin{abstract}
Objective: To determine frequency of early postoperative cognitive dysfunction (POCD) in patients aged 40 to 60 years, following general anaesthesia in patients undergoing elective, non-cardiac surgery.

Study Design: Descriptive study.

Place and Duration of Study: Department of Anaesthesiology, The Aga Khan University Hospital (AKUH), Karachi, from December 2015 to May 2016.

Methodology: After obtaining approval from Ethical Review Committee of AKUH and informed consent, ASA I and II patients, aged between 40 to 60 years of either gender, undergoing general anaesthesia for elective non-cardiac surgical procedures, were recruited. Patients were assessed preoperatively by using mini-mental state examination (MMSE); and patients having a score equal to or greater than 23 (maximum 30) were included in the study. All patients were reassessed at 24 hours postoperatively by MMSE. Both the MMSE evaluations were performed by primary investigator on predesigned data collection form.

Results: A total of 150 patients were enrolled in the study. Preoperative MMSE score ranged from 24 to 30 while postoperative MMSE score (at 24 hours) was between 25 and 30. Thus, no patient developed POCD following general anaesthesia for elective, non-cardiac surgery in this study.

Conclusion: Early POCD was not found in the presently studied population of middle aged patients having elective non-cardiac surgery under general anaesthesia.
\end{abstract}

Key Words: Postoperative cognitive dysfunction (POCD), General anaesthesia, Non-cardiac surgery, Mini- mental state examination.

How to cite this article: Yousuf MS, Samad K, Ullah H. Postoperative Cognitive Dysfunction following General Anaesthesia in Patients Undergoing Elective Non-cardiac Surgery. J Coll Physicians Surg Pak 2020; 30(04):417-419. DOI: https://doi.org/10.29271/jcpsp.2020.04.417.

\section{INTRODUCTION}

Cognitive dysfunction is an impairment of cognitive functions which include memory, learning, concentration, and speed of mental processing. ${ }^{1}$ It is more common in the elderly population with the incidence of upto $47 \%$ at 24 hours after minor procedure. ${ }^{2}$ Cognitive functions are frequently affected postoperatively and post-anaesthesia in elderly populations. Some studies had found that there is higher incidence of POCD in patients having pre-existing coronary atherosclerosis. ${ }^{3}$ The two main risk factors which cause POCD include inflammatory response and the use of opioids. ${ }^{4}$ The consequences of chronic POCD in middle-aged patients are catastrophic, as it especially affects routine daily activities. The aim of this study was to investigate the frequency of early POCD in patients aged 40 to 60 years, after elective, non-cardiac surgery. The data regarding the POCD in patients aged 40 to 60 years is very limited, so determining the frequency and its correlates will help address the risk factors in middle aged patients as well.

Correspondence to: Dr. Muhammad Saad Yousuf, Department of Anaesthesiology, The Aga Khan University Hospital, P.O. Box. 3500, Stadium Road, Karachi-74800, Pakistan E-mail:saad.yousuf@aku.edu

Received: February 19, 2019; Revised: August 16, 2019;

Accepted: September 16, 2019

DOI: https://doi.org/10.29271/jcpsp.2020.04.417

\section{METHODOLOGY}

After obtaining approval from the Ethical Review Committee and Institutional Anaesthesia Review Committee from December 2015 to May 2016. This descriptive study was prospectively conducted in preoperative area and wards of Aga Khan University Hospital, Karachi.

Based on study done by Rohan et al., which reported a $47 \%$ incidence of POCD, ${ }^{2}$ at 24 hours and taking $10 \%$ margin of error, 150 patients were included in this study by using nonprobability, consecutive sampling technique.

After obtaining the informed written consents, inpatients of either gender aged from 40 to 60 years and undergoing elective non-cardiac surgeries of 2 - 4 hours duration requiring general anaesthesia, were included in the study. All recruited patients were status 1 and 2 of American Society ofAnesthesiologists(ASA), with preoperative MMSE score above 23. Patients having neurological, carotid and cardiac surgeries, with central nervous system disorders including pre-existing cognitive dysfunction as mini-mental score examination (MMSE) score less than 23, cerebrovascular diseases, drug dependence (consumption of tranquilisers or antidepressants, anti-psychotic, benzodiazepines and opioids), visual or auditory disorders, and neuraxial block were excluded from the study. Patients having multiple surgeries during same admission were included in the study only once. 
All included patients were reassessed by MMSE at 24 hours postoperatively. POCD was defined as MMSE score of less than 23 postoperatively in patients with MMSE score above 23 in the preoperative evaluation. Preoperative and postoperative assessments were made by primary investigator on predesigned data collection form.

Statistical packages for social science version 19 (SPSS Inc., Chicago, IL) was used for statistical analysis. Categorical variables like incidence of POCD, sex and ASA status, were calculated as frequencies and percentages. Quantitative variables like age, weight, height, duration of surgery, duration of anaesthesia and MMSE score were calculated as mean and standard deviation. A pvalue of less than 0.05 was considered as level of significance. Stratification analysis was planned based on the occurrence of POCD.

\section{RESULTS}

A total of 150 patients, undergoing elective non-cardiac surgery under general anaesthesia, were included in this study. The mean age of patients was $48.96 \pm 6.27$ years with majority of them being females. Regarding body mass index, 4 patients were underweight, $38.67 \%(n=58)$ were overweight, and $35.33 \%(n=53)$ were obese (Table I). Out of the 150 recruited patients, 32 were ASA-I and 118 were ASA-II patients. Out of 150 cases, hypertension was observed in $30.67 \%$ and diabetes mellitus was found in $18.67 \%$.

Table I: Demographic and duration of anaesthesia and surgery statistics of the patients $(\mathrm{N}=150)$.

\begin{tabular}{|l|c|c|c|c|}
\hline Variables & Mean & $\begin{array}{c}\text { Std. } \\
\text { deviation }\end{array}$ & Median & $\begin{array}{c}\text { Interquartile } \\
\text { range }\end{array}$ \\
\hline Age (years & 48.96 & 6.27 & 48 & 11 \\
\hline Weight (kg) & 70.55 & 13.22 & 70 & 18 \\
\hline Height (cm) & 157.48 & 7.94 & 157 & 9 \\
\hline BMI (kg/m ${ }^{2}$ ) & 28.52 & 5.35 & 28.16 & 7.9 \\
\hline $\begin{array}{l}\text { Duration of anaesthesia } \\
\text { (minutes) }\end{array}$ & 189 & 110 & 169 & 121 \\
\hline $\begin{array}{l}\text { Duration of surgery } \\
\text { (minutes) }\end{array}$ & 163 & 94 & 126 & 104 \\
\hline
\end{tabular}

Overall, the median preoperative MMSE score was 30, while the median postoperative MMSE score was 29 (Figure 1). POCD was not observed in this study. Stratification analysis was not performed because no case of POCD was observed in the study.

\section{DISCUSSION}

POCD is common in elderly patients as compared to middle-aged patients. Until now, many studies have been done in which patients undergoing major surgery were included; and the main focus was on long-term postoperative cognitive dysfunction. ${ }^{5}$ Only few studies are available in which minor procedures, mainly as out-patients, were done to investigate POCD few days after the procedure ${ }^{6}$ and compared different anaesthetic techniques. ${ }^{7}$ The authors included only middle-aged patients, who underwent elective non-cardiac surgeries and then estimated the incidence of POCD.

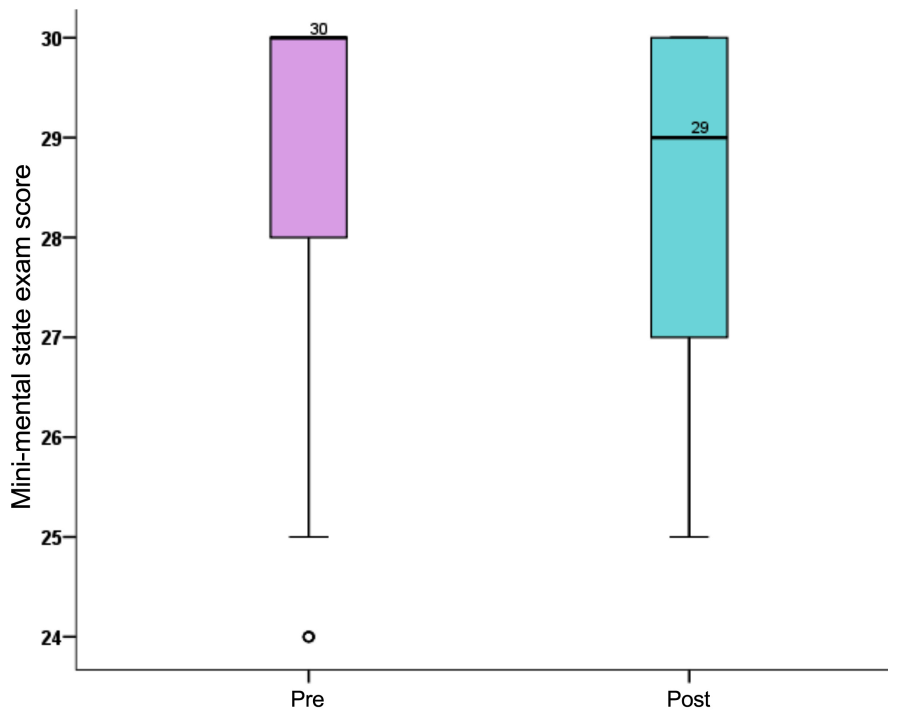

Figure 1: Preand postmedian mini-mental state exam score $(\mathrm{N}=150)$.

This study investigated the effect of general anaesthesia in middle-age patients. All patients, who required general anaesthesia for elective non-cardiac procedures having aged between 40 and 60 years with physical ASA status of I or II, were recruited in this study. Patients were assessed in the preoperative area by using MMSE. All patients having MMSE score above 23 were then included in the study. These patients were reassessed at 24 hours postoperatively. The POCD was not observed in this study. Rohan etal. concluded that POCD was $47 \%$ regardless of general anaesthesia technique. ${ }^{2}$ However, this study's demographics differ in that the patient were middle-aged (mean age 48 years in contrast to 73 years).

In elderly patients (aged between 60 and 81 years), an international trial was conducted in non-cardiac surgeries, and observed POCD with an incidence of $26 \%$ and $10 \%$ at one week and three months, respectively. ${ }^{8}$ While in middle-aged patients (aged between 40 and 60 years), the incidence of POCD was $19 \%$ and $6 \%$ at one week and three months, respectively. ${ }^{9}$ The main limitation of these studies were that they did not compare the incidence of POCD, observed with the extent of major surgery and general anaesthesia.

Moller et al. found that the risk factors for early POCD include advanced age, prolong anaesthesia, less education, more than one operation and postoperative respiratory dysfunctions; but only age was found to be a risk factor for late POCD. ${ }^{10}$ Boos et al. observed the POCD in $14.54 \%$ patients at 24 hours postoperatively with overall prevalence of $29 \%$. All POCD cases were observed in patients above 65 years of age. ${ }^{11}$ Bedford found no evidence of long-term postoperative cognitive dysfunction after general surgery. ${ }^{12}$ Small sample size and inappropriate control group were the main limitations of this study.

The correlation of early POCD with epidural analgesia is found to be difficult. It has been expected that the incidence of POCD might be reduced by utilizing only regional anaesthesia, but in contrast the findings were opposite in middle-aged patients. The suggestion behind this was increase plasma concentration of bupivacaine that mightcause acute neurologic toxicity. ${ }^{13}$ 
However, in one study it was found that the incidence of POCD after regional anaesthesia decreased at seven days after non-cardiac surgeries when compared with general anaesthesia. ${ }^{14}$

Considering information obtained from studies done in the past, POCD has been categorised in acute, intermittent and chronic changes. Acute POCD was described as cognitive decline observed within one week post-procedure; intermediate POCD was observed in less than 3 months while the long-term POCD was observed from one-to-two years post-procedure. Although, the relevance of investigating POCD during these time-frame is not clear. The clinical significance of time duration, at which a diagnosis of POCD been made, is not yet identified. Moreover, there is no study has been conducted that found the relevance of carrying out assessments at a particular time-frame. ${ }^{15} \mathrm{POCD}$ is a common complication after cardiac surgery. Newman et al. found that procedures requiring cardiopulmonary bypass increases the incidence of POCD to as high as approximately $50 \%$ at discharge. ${ }^{5}$

In one study, it was found that the health-related complaints were associated with the total number of surgeries and the extent of anaesthesia; but it did not predict cognitive behavior. ${ }^{8}$

In many other studies, the assessments were done for more than 6 months post-surgery, but they didn't find any significant change from that measured preoperatively. ${ }^{16} \mathrm{~A}$ recent study concluded that the increase in postoperative mortality was associated with acute POCD. ${ }^{17}$ Therefore, a conclusive study is required which explains the prognostic importance of cognitive dysfunction related to procedure and hospitalisation, impact of surgery on the quality of life and cognition, and also to anticipate the modifiable riskfactors.

\section{CONCLUSION}

POCD is one of the clinical conditions which has multifactorial origin. In our study, middle-aged patients (40 - 60 years of age), some of them having diabetes and/or hypertension and undergoing upto 4 hours of elective, non-cardiac surgery under general anaesthesia, did not develop POCD on MMSE evaluation done 24 hours after the surgery. Further research is required to investigate other risk factors in the causation of POCD in middle-aged population.

ETHICAL APPROVAL: Study was conducted after obtaining approval from the Ethical Review Committee of AKUH.

\section{PATIENTS' CONSENT:}

Consent for the participation in study was taken from patients.

\section{CONFLICT OF INTEREST:}

Authors declared no conflict of interest.

\section{AUTHORS' CONTRIBUTION}

MSY, KS, HU: Data collection, analysis, and literature review. All authors approved final version of the manuscript.

\section{REFERENCES}

1. Monk TG, Weldon BC, Garvan CW. Predictors of cognitive dysfunction after major noncardiac surgery. Anesthesiology 2008; 108:18-30.

2. Rohan D, Buggy D, Crowley S, Ling FKH. Increased incidence of postoperative cognitive dysfunction $24 \mathrm{hr}$ after minor surgery in the elderly. Can J Anaesth 2005; 52:137-142.

3. Kline RP, Pirraglia E, Cheng H, De Santi S, Li Y, Haile M, et al. Surgery and brain atrophy in cognitively normal elderly subjects and subjects diagnosed with mild cognitive impairment. Anesthesiology 2012; 116:603-12.

4. Pether KJ, Jan LH, Narinder. Does depth of anaesthesia influence postoperative cognitive dysfunction or inflammatory response following major ent surgery. J Anesth Clin Res 2012; 3:6.

5. Newman MF, Kirchner JL, Phillips-Bute B, Gaver V, Grocott H, Jones $\mathrm{RH}$, et al. Longitudinal assessment of neurocognitive function after coronary artery bypass surgery. N Engl J Med 2001; 344:395-402.

6. Tzabar Y, Asbury AJ, Millar K. Cognitive failures after generalan aesthesia for day-case surgery. $\mathrm{Br}$ J Anaesth 1996; 76:194-7.

7. Heath PJ, Ogg TW, Gilks WR. Recovery after day-case anaesthesia a 24-hour comparison of recovery after thiopentoneor propofol anaesthesia. Anaesthesia 1990; 45:911-15.

8. Dijkstra JB, Van Boxtel MP, Houx PJ, Jolles J. An operation under general anaesthesia as a risk factor for age-related cognitive decline results from a large cross-sectional population study. J Am Geriatr Soc 1998; 46:1258-65.

9. Johnson T, Monk T, Rasmussen LS, Abildstrom H, Houx $P$, Korttila $\mathrm{K}$, et al. Postoperative cognitive dysfunction in middle-aged patients. Anesthesiology 2002; 96:1351-7.

10. Moller JT, Cluitmans P, Rasmussen LS, Houx P, Rasmussen $H$, Canet J, et al. Long-term postoperative cognitive dysfunction in the elderly ISPOCD1 study. Lancet 1998; 351:857-61.

11. Boos GL, Soares LF, Oliveira Filho GR. Postoperative cognitive dysfunction prevalence and associated factors. Rev Bras Anestesiol 2005; 55:517-24.

12. Bedford PD. Adverse cerebral effects of anaesthesia on old people. Lancet 1955; 266:259-64.

13. Emanuelsson BM, Zaric D, Nydahl PA, Axelsson KH. Pharmacokinetics of ropivacaine and bupivacaine during 21 hours of continuous epidural infusion in healthy male volunteers. Anesth Analg 1995; 81:1163-8.

14. Rasmussen LS, Johnson T, Kuipers HM, Kristensen D, Siersma VD, Vila $P$, et al. Does anaesthesia cause postoperative cognitive dysfunction a randomised study of regional versus general anaesthesia in 438 elderly patients. Acta Anaesthesiol Scand 2003; 47:260-6.

15. Tsai TL, Sands LP, Leung JM. An update on postoperative cognitive dysfunction. Adv Anesth 2010; 28:269-84.

16. Abildstrom $H$, Rasmussen LS, Rentowl $P$, Hanning $C D$, Rasmussen $\mathrm{H}$, Kristensen PA, et al. Cognitive dysfunction 1-2years after non-cardiac surgery in the elderly. Acta Anaesthesiol Scand 2000; 44:1246-51.

17. Steinmetz J, Christensen KB, Lund T, Lohse N, Rasmussen LS. Ispocd group. Long term consequences of postoperative cognitive dysfunction. Anesthesiology 2009; 110:548-55. 